\title{
Tomografia computadorizada aplicada a estudos de um Planossolo(1)
}

\author{
Alceu Pedrotti( ${ }^{(2)}$, Eloy Antonio Pauletto ${ }^{(3)}$, Silvio Crestana ${ }^{(4)}$, Paulo Estevão Cruvinel(4), \\ Carlos Manoel Pedro Vaz ${ }^{(4)}$, João de Mendonça Naime ${ }^{(4)}$ e Alvaro Macedo da Silva ${ }^{(4)}$
}

Resumo - Na busca de técnicas mais apuradas para a determinação e avaliação de parâmetros físicos do solo com aplicabilidade em várzeas, vem se destacando a tomografia computadorizada, por medir a densidade e a umidade com boa sensibilidade e alta resolução espacial. O presente trabalho teve como objetivo descrever aspectos e procedimentos da calibração de um minitomógrafo de raios-X e gama para estudo da densidade e umidade de um Planossolo no Rio Grande do Sul, bem como estabelecer parâmetros estatísticos para sua adequada utilização. A calibração do minitomógrafo foi obtida pela regressão linear entre as unidades tomográficas (UT), apresentadas pelo programa de reconstrução de imagem, e os coeficientes de atenuação linear $\left(\mu_{1}, \mathrm{~cm}^{-1}\right)$, medidos por transmissão direta de raios gama, em amostras dos horizontes A e B do Planossolo, água destilada, benzina e alumínio. Para as medidas de transmissão direta de radiação utilizaram-se recipientes com água destilada, benzina, solo e Al, obtendo-se as seguintes fórmulas para o cálculo da densidade do solo no horizonte $A: D_{s}=[(U T / 986,16)-(0,200 x \theta)] /$ 0,267; e no horizonte $\mathrm{B}: \mathrm{D}_{\mathrm{s}}=[(\mathrm{UT} / 986,16)-(0,200 \times \theta)] / 0,297$, em que UT é o valor médio de UT em cada linha e $\theta$ é a umidade volumétrica da amostra de solo, em $\mathrm{m}^{3} \mathrm{~m}^{-3}$. Com as configurações obtidas, verificou-se variabilidade média de $2,74 \%$ e $0,73 \%$, respectivamente, em termos de homogeneidade e repetibilidade. Os erros atribuídos ao equipamento são de 0,051 e $0,046 \mathrm{Mg} \mathrm{m}^{-3}$, respectivamente, nos horizontes Ae B, revelando precisão e adaptabilidade no emprego da técnica em estudos do Planossolo.

Termos para indexação: várzea, densidade do solo, umidade do solo, calibração, minitomógrafo.

\section{Computer-assisted tomography for studies of an Albaqualf}

Abstract - In order to find better techniques to evaluate the soil physical parameters applied to lowland soils, the computerized tomography has been used to measure soil density and water content with accuracy and high spatial resolution. This work was carried out in order to describe features and calibration procedures of a computerized minitomographer using X-ray and gama-rays as sources of radiation and to establish suitable statistical parameters on the study of soil bulk density and water content in a Planosol (Albaqualf) from Rio Grande do Sul State, Brazil. The minitomographer calibration was obtained from the linear regression equation among the tomography's unities (TU) presented by the image reconstruction program and the linear attenuation coefficient $\left(\mu_{1}, \mathrm{~cm}^{-1}\right)$, by the measurement of direct transmission of $\gamma$-rays as source of radiation in soil samples from A and B horizons, distilled water, benzin and aluminum. In order to get measures of the direct radiation transmission, containers with distilled water, benzin, soil and aluminum were used to obtain the following equations to calculate soil bulk density in the A horizon: $\mathrm{D}_{\mathrm{s}}=[(\mathrm{TU} / 986.16)-(0.200 \times \theta)] / 0.267$ and in the $\mathrm{B}$ horizon: $\mathrm{D}_{\mathrm{s}}=[(\mathrm{TU} / 986.16)-(0.200 \mathrm{x} \theta)] / 0.297$, where TU is the mean value in the line and $\theta$ is the soil volumetric water content $\left(\mathrm{m}^{3} \mathrm{~m}^{-3}\right)$. The obtained configurations allowed to attain average variabilities of $2.74 \%$ and $0.73 \%$ for homogeneity and repeatability, respectively. The expected errors related to the equipment are 0.051 and $0.046 \mathrm{Mg} \mathrm{m}^{-3}$, to the $\mathrm{A}$ and $\mathrm{B}$ horizons, respectively. The results showed the technique accuracy and adaptability in the studies of the physical characteristics of a Planosol.

Index words: lowland, soil density, soil moisture, calibration, miniscanner.

(1) Aceito para publicação em 4 de abril de 2003 .

(2) Universidade Federal de Sergipe, Dep. de Engenharia Agronômica, Av. Mal. Rondon, s/no, Campus Universitário Jardim Rosa Elze, CEP 49100-000 São Cristóvão, SE. E-mail: apedroti@ufs.br

(3) Universidade Federal de Pelotas, Fac. de Agronomia Eliseu
Maciel, Dep. de Solos, Caixa Postal 354, CEP 96001-970 Pelotas, RS. E-mail: pauletto@ufpel.tche.br

(4) Embrapa-Centro Nacional de Pesquisa e Desenvolvimento de Instrumentação Agropecuária, Caixa Postal 741, CEP 13560-970 São Carlos, SP. E-mail: crestana@cnpdia.embrapa.br, cruvinel@cnpdia.embrapa.br,vaz@cnpdia.embrapa.br, naime@cnpdia.embrapa.br, alvaro@cnpdia.embrapa.br 


\section{Introdução}

No Rio Grande do Sul, os solos de várzea ocupam aproximadamente $22,85 \%$ da área total, sendo encontrados nas regiões fisiográficas da Planície Costeira, Depressão Central e Campanha. Os Planossolos ocupam cerca de $56 \%$ da área total dos solos hidromórficos, representando cerca de $11 \%$ da área do Estado. Essa classe é representada pelos Planossolos Hidromórficos eutróficos típico e solódico, e pelos Planossolos Háplicos eutróficos típico e vértico (Embrapa, 1999). Por causa da heterogeneidade do material de origem e dos diferentes graus de hidromorfismo, apresentam grande variação nas características morfológicas, físicas, químicas e mineralógicas, fazendo com que sejam agrupados em diferentes classes, com diferentes limitações e aptidões de uso (Pinto et al., 1999).

Segundo Pinto et al. (1999), a utilização dos solos hidromórficos mediante sistemas agrícolas alternativos e incorporação de culturas de sequeiro, de maneira mais racional, pode ser viabilizada pelo melhoramento genético, para obtenção de cultivares de espécies alternativas melhor adaptadas às condições do meio, ou por um manejo adequado do solo. O melhoramento genético propicia soluções mais definitivas, embora necessite de prazos mais longos; já o manejo do solo, se realizado de forma racional, poderá proporcionar resultados favoráveis em menores prazos.

Para a utilização de culturas alternativas, como soja, milho e pastagem, em rotação com a cultura do arroz irrigado, torna-se necessário um manejo hídrico diferente do atualmente usado na lavoura arrozeira, associado a um manejo de solo adequado para cada espécie a ser introduzida, razão pela qual os atributos físicos e hídricos devem ser quantificados para a condução dessas lavouras (Pedrotti, 1996).

Embora se venha buscando melhores alternativas de utilização dos solos de várzea, as medidas para caracterização física, feitas para se definir os melhores sistemas de cultivo, esbarram em métodos tradicionalmente utilizados que nem sempre são os mais adequados e eficientes (Pedrotti, 1996).

Entre as novas técnicas destaca-se a tomografia computadorizada (TC) de raios-X e gama (Cruvinel \& Colnago, 2000), porque não perturba a estrutura natural das amostras e porque apresenta sensibilidade e alta resolução espacial. Além do valor médio, no caso de densidade e de umidade do solo, a TC indica a localização exata das possíveis heterogeneidades presentes internamente e os valores extremos, pois desconsidera a isotropia espacial. As desvantagens deste método são o alto custo e a demora para obtenção dos dados, principalmente em resoluções espaciais maiores (Cruvinel et al., 1990; Crestana, 1992; Crestana et al., 1992, 1996; Cássaro, 1994; Cruvinel \& Colnago, 2000).

A TC por transmissão de raios-X e gama é um processo de obtenção de imagem reconstruída da seção transversal ou fatia de um corpo, em um computador, utilizando-se um equipamento externo ao mesmo, o tomógrafo (Appoloni \& Cesareo, 1994). Uma descrição detalhada da tomografia computadorizada, com relação a seus princípios, aplicabilidade, potencialidades, operacionalização e metodologia empregada, métodos de reconstrução de imagem tomográfica e radiação nuclear pode ser encontrada em Ferraz \& Mansell (1979), Crestana (1985), Cruvinel (1987), Vaz (1989), Aylmore (1993), Anderson \& Hopmans (1994), Naime (1994), Pedrotti (1996) e Cruvinel \& Colnago (2000).

A TC pode ser utilizada para medir a densidade, a porosidade e a umidade de amostras de solos de formas irregulares (Pedrotti, 1996), ou seja, avaliar o nível de compactação; para isto necessita da determinação dos parâmetros acima mencionados de cada solo, para calibração e avaliação de desempenho do equipamento, por meio de parâmetros estatísticos (Pedrotti, 1996; Cruvinel \& Colnago, 2000).

O objetivo deste trabalho foi descrever os aspectos e os procedimentos da calibração de um minitomógrafo de raios-X e gama para estudo da densidade e umidade de um Planossolo do Rio Grande do Sul, bem como estabelecer parâmetros para sua adequada utilização.

\section{Material e Métodos}

As amostras de solo foram coletadas na EmbrapaCentro de Pesquisa Agropecuária de Clima Temperado, Estação Experimental de Terras Baixas (EETB), Município de Capão do Leão, RS. O solo foi classificado como Planossolo Hidromórfico eutrófico solódico (Embrapa, 
1999; Pinto et al., 1999), correspondente a Planossolo solódico (IBGE, 1986), com argila de atividade alta, textura média argilosa com relevo plano (Pedrotti et al., 2001), e como Albaqualf segundo Estados Unidos (1990). Foram empregadas amostras indeformadas - coletadas em cilindros de PVC com $100 \mathrm{~mm}$ de diâmetro e $30 \mathrm{~cm}$ de comprimento, pelo método hidraúlico de amostragem indeformada de solo (MHAIS) (Pedrotti et al., 2001) - e amostras deformadas, utilizadas na calibração do minitomógrafo. As análises tomográficas e a obtenção das imagens por transmissão de raios gama nas amostras de solo foram realizadas com o minitomógrafo computadorizado de raios- $X$ e gama, da Embrapa-Centro Nacional de Pesquisa e Desenvolvimento de Instrumentação Agropecuária, em São Carlos, SP. As amostras indeformadas foram seccionadas a cada $7,50 \mathrm{~cm}$, vedadas com parafina, e foram marcadas as suas respectivas orientações espaciais ao longo do perfil do solo.

O minitomógrafo é composto de um sistema fontedetector, constituído de uma fonte radioativa de raios gama ${ }^{241} \mathrm{Am}$, energia $59,6 \mathrm{keV}$ e atividade $300 \mathrm{mCi}\left(37 \times 10^{8} \mathrm{~Bq}\right)$; detector de cristal cintilador $\mathrm{NaI}(\mathrm{Tl})$ de 3"x3" acoplado a uma válvula fotomultiplicadora com tensão de polarização de $760 \mathrm{~V}$; preamplificador ORTEC modelo 376; amplificador IPEN-CPIO-AIE modelo PM321; discriminador e contador; computador para controle do mecanismo de varredura, aquisição dos dados e reconstrução matemática da imagem; e sistema mecânico de varredura tomográfica de 1a geração (Cruvinel, 1987). $\mathrm{Na}$ obtenção de feixes finos de fótons, foram utilizadas na fonte e no detector colimadores de chumbo do tipo circular e com diâmetros de abertura opcionais. Foram usados colimadores com orifício circular de $4 \mathrm{~mm}$ de diâmetro e 20 mm de comprimento (Crestana, 1985; Cruvinel, 1987; Cruvinel et al., 1990; Cruvinel \& Colnago, 2000). O diagrama de blocos do equipamento utilizado no presente estudo está apresentado na Figura 1.

A calibração do minitomógrafo foi feita por meio de regressão linear entre as unidades tomográficas (UT) obtidas pelo programa de reconstrução de imagem e os coeficientes de atenuação linear $\mu_{1}\left(\mathrm{~cm}^{-1}\right)$, medidos por transmissão direta, nos seguintes materiais: amostras deformadas de Planossolo (horizontes Ae B), água destilada, benzina e Al corpo de prova. Utilizou-se terra fina secada em estufa (TFSE), passada em peneira (malha de 1,0 mm), acondicionada em recipientes de dimensões conhecidas, para se encontrar o coeficiente de atenuação linear $\left(\mu_{1}\right)$. Realizaram-se, no mínimo, 20 leituras em cada condição de feixe livre (recipiente vazio) e com material ou corpo de prova.

O cálculo do coeficiente de atenuação linear, por meio da equação de Lambert-Beer foi feito medindo-se o número de fótons transmitidos através do recipiente vazio ou do ar $\left(\mathrm{I}_{\mathrm{o}}\right)$ e o número de fótons transmitidos através do recipiente com amostra ou do corpo de prova (I) e a espessura $(\mathrm{x}, \mathrm{cm})$ que o feixe percorre no interior da amostra:

$\mu_{1}=\frac{1}{\mathrm{x}} \cdot \ln \left(\frac{\mathrm{I}_{0}}{\mathrm{I}}\right)$.

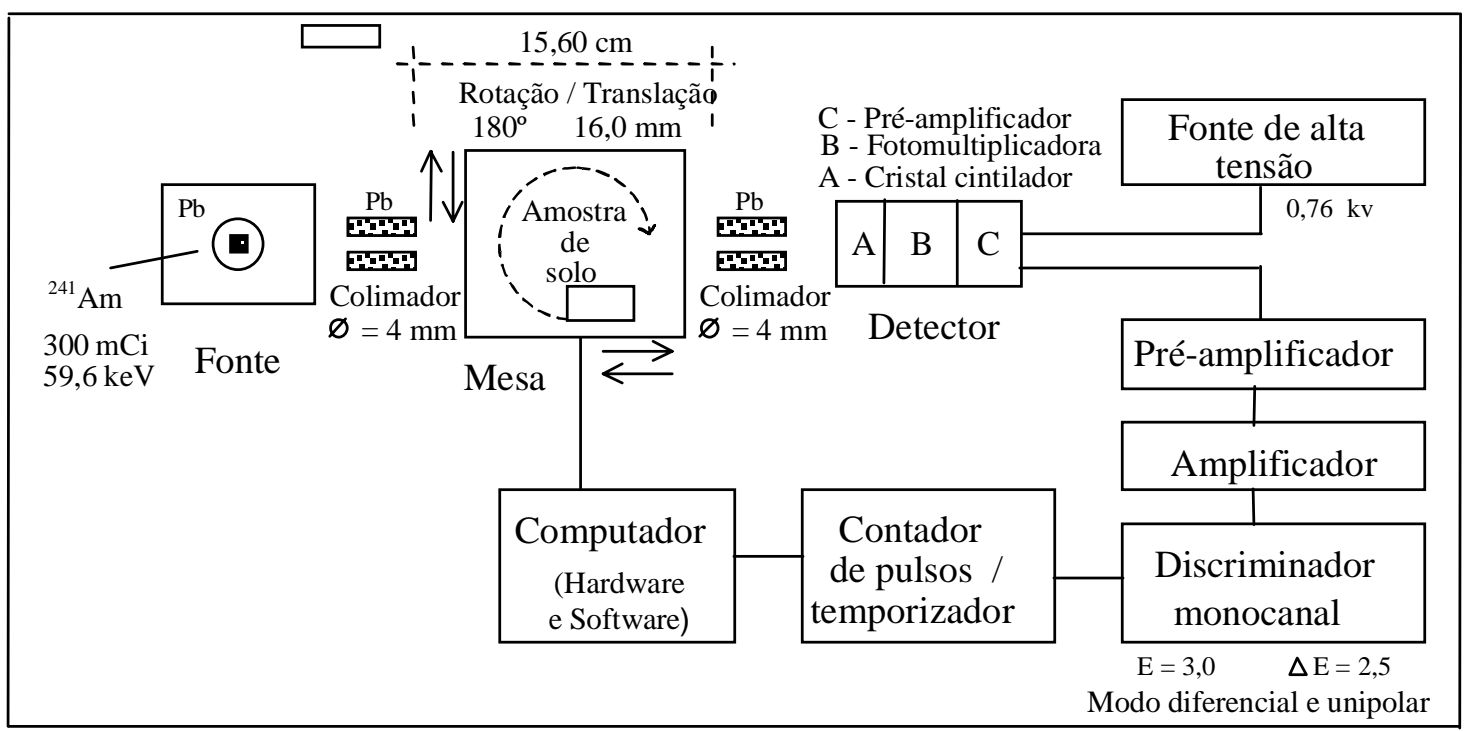

Figura 1. Diagrama de blocos do minitomógrafo de raios-X e gama. 
O tempo de contagem foi de 10 segundos, com 20 repetições em cada amostra.

Os coeficientes de atenuação linear obtidos em uma tomografia são diretamente proporcionais ao grau de adensamento/compactação (ou densidade, $\rho$ ) em cada ponto da seção transversal da amostra. O sinal negativo indica o comportamento inversamente proporcional da intensidade do feixe de radiação gama emergente em relação à espessura da amostra de solo, isto é, quando dx aumenta, I diminui. Um horizonte de um dado tipo de solo é caracterizado pelo coeficiente de atenuação em massa $\left(\mu_{\mathrm{m}}, \mathrm{cm}^{2} / \mathrm{g}\right)$ :

$\mu_{\mathrm{m}}=\mu_{1} / \rho$.

Para determinação do coeficiente de atenuação em massa, o solo foi peneirado em malha de $1 \mathrm{~mm}$ e secado em estufa $\left(105^{\circ} \mathrm{C}\right.$ por 48 horas), calculando-se a densidade pelo método gravimétrico e medindo-se $\mu_{1}$ por transmissão de raios gama:

$\mu_{1}=\mu_{\mathrm{m}-\mathrm{s}} \cdot \rho+\mu_{\mathrm{m}-1} \cdot \theta$,

em que: $\mu_{1}\left(\mathrm{~cm}^{-1}\right)$ é o coeficiente de atenuação linear do solo com umidade $\theta ; \mu_{\mathrm{m}-\mathrm{s}}\left(\mathrm{cm}^{2} \mathrm{~g}^{-1}\right)$ é o coeficiente de atenuação em massa do material (solo); $\rho\left(\mathrm{g} \mathrm{cm}^{-3}\right)$ é a densidade do solo; $\mu_{\mathrm{m}-1}\left(\mathrm{~cm}^{2} \mathrm{~g}^{-1}\right)$ é o coeficiente de atenuação em massa do líquido (água) e $\theta\left(\mathrm{m}^{3} \mathrm{~m}^{-3}\right)$ é a umidade volumétrica do solo (Pedrotti, 1996).

A equação de calibração do minitomógrafo foi determinada mediante regressão linear entre os $\mu_{1}$ determinados por transmissão direta e as UTs produzidas pelo programa de reconstrução, com os diferentes materiais. Assim, quando se deseja saber o $\mu_{1}$ de um dado pixel, para a energia de 59,6 keV, é suficiente fazer a divisão da UT pelo coeficiente de calibração (coeficiente angular da reta de calibração do minitomógrafo).

Os parâmetros estatísticos utilizados para avaliar a calibração do equipamento foram a repetibilidade, homogeneidade, sensibilidade e os erros inerentes à técnica, em virtude do caráter estatístico da emissão de radiação e à instrumentação utilizada. Para verificação da homogeneidade e repetibilidade dos dados obtidos pela $\mathrm{TC}$, foram feitas três tomografias distintas de uma substância homogênea (água destilada) nas mesmas condições de ajuste do equipamento e em horários diferentes quanto ao início da aquisição dos dados. Analisou-se estatisticamente, comparando-se os valores da média, moda, desvio-padrão, coeficiente de variação e variância. Determinou-se também a sensibilidade do equipamento fazendo-se variar de uma unidade a UT para um valor médio constante da umidade volumétrica da amostra. O erro atribuído ao equipamento foi determinado aplicandose o coeficiente de variação obtido com uma substância homogênea (água destilada) à média dos valores de UT das amostras analisadas.
Na quantificação da densidade pela tomografia computadorizada, primeiramente escolheu-se o plano de interesse na amostra e, pela operacionalização do aparelho em si, procedeu-se a realização da tomografia propriamente dita. Os parâmetros de varredura tomográfica utilizados foram o deslocamento linear de $16,00 \mathrm{~cm}$, passo linear de $0,40 \mathrm{~cm}$, rotação total de $180^{\circ}$, passo angular de $4,50^{\circ}$, tempo de contagem de 10 segundos e número de fótons do feixe livre aproximadamente igual a 20,2 mil. A partir da matriz de dados de 40x40 contagens, foram reconstruídas as imagens tomográficas. Os parâmetros descritos acima são variáveis com a energia da radiação e com a dimensão e densidade das amostras analisadas. $\mathrm{O}$ tempo total de varredura tomográfica foi de aproximadamente 10 horas e 46 minutos.

A imagem tomográfica foi reconstruída pelo programa de reconstrução de imagem, pelo método da retroprojeção filtrada (Cruvinel, 1987). Essas imagens representam um mapa de valores de UTs calculados para cada pixel (4 mm x $4 \mathrm{~mm}$ ), visualizados por atribuição de uma escala de 16 tons de cinza, em que o preto corresponde à menor UT e o branco à maior UT.

\section{Resultados e Discussão}

Os valores dos desvios-padrões são relativamente baixos, o que demonstra a precisão das medidas obtidas (Tabela 1). Aylmore (1993) cita vários trabalhos cujas amplitudes de valores dos desviospadrões são semelhantes às encontradas no presente experimento. As pequenas divergências são atribuídas aos próprios materiais, por não serem totalmente puros, aos possíveis erros experimentais e às diferentes técnicas de reconstrução de imagem.

Tabela 1. Valores médios obtidos do coeficiente de atenuação em massa $\left(\mu_{\mathrm{m}}\right)$ determinados com o minitomógrafo de raios gama, para a energia de $59,6 \mathrm{keV}$ e valores encontrados na literatura com sua respectiva referência.

\begin{tabular}{lll}
\hline Material & \multicolumn{2}{c}{ Coeficiente de atenuação em massa $\left(\mathrm{cm}^{2} \mathrm{~g}^{-1}\right)$} \\
\cline { 2 - 3 } & \multicolumn{1}{c}{ Obtido } & \multicolumn{1}{c}{ Literatura } \\
\hline Água destilada & $0,1999 \pm 0,002^{(1)}$ & 0,2049 (Corey et al., 1971) \\
& & 0,2012 (Mansel et al., 1973) \\
& & 0,2006 (Ferraz, 1974) \\
& & 0,2000 (Vaz, 1989) \\
& & 0,2034 (Chieppe Junior, 1993) \\
& & 0,2210 (Cássaro, 1994) \\
\hline Alumínio & $0,7506 \pm 0,030^{(2)}$ & $0,7550 \pm 0,0040$ (Vaz, 1989) \\
\hline Benzina & $0,1250 \pm 0,002^{(2)}$ & $0,1370 \pm 0,0008$ (Cássaro, 1994) \\
\hline Planossolo - horizonte A & $0,2670 \pm 0,009^{(2)}$ & \\
Planossolo - horizonte B & $0,2974 \pm 0,010^{(2)}$ & \\
\hline \multirow{2}{*}{ (1)Média de 30 repetições. ${ }^{(2)}$ Média de 20 repetições. }
\end{tabular}


Esses resultados podem ser considerados bons e são atribuídos à boa colimação do feixe emergente com o detector, à determinação com "janela" de energia estreita, à alta taxa de contagem de fótons (feixe livre igual a 20.200 contagens por segundo e feixe atenuado, com amostra igual a 2.500 contagens por segundo) e aos $\mu_{1} \mathrm{~s}$ próximos dos valores ideais ou mesmo dos valores teóricos, como 0,2052 para a água, conforme Ferraz (1974). Tal comportamento atende à proposição de parâmetros ideais para uma condição tomográfica ótima, feita por Appoloni \& Cesareo (1994).

O horizonte A do Planossolo apresentou coeficiente de atenuação em massa menor que o horizonte $\mathrm{B}$, provavelmente por causa dos maiores teores de caulinita, silício e óxidos de $\mathrm{Al}$ (ricos em $\mathrm{Al}$ ) encontrados neste horizonte. Já o horizonte B apresentou maiores valores médios, provavelmente em virtude do maior teor de argila (maior quantidade relativa de óxidos de $\mathrm{Fe}$ e $\mathrm{Al}$, em relação ao horizonte A) (Pedrotti et al., 2001), esta com valores de coeficientes elevados (Ferraz \& Mansell, 1979; Aylmore, 1993). Segundo Vaz (1989), é comum encontrar variações de 0,25 a $0,43 \mathrm{~cm}^{2} \mathrm{~g}^{-1}$, quando se determina o $\mu_{\mathrm{m}}$ de diferentes tipos de solos. Esses coeficientes são dependentes da composição química do material quando se usa energia semelhante à utilizada no presente estudo (Ferraz \& Mansell, 1979).

$\mathrm{O}$ valor teórico ideal do $\mu_{1}$ para a espessura da amostra adotada neste trabalho $(7,5 \mathrm{~cm})$ foi de 0,25 por $\mathrm{cm}$, significando que os valores encontrados são relativamente próximos do ideal para o solo em estudo. Ferraz (1974) ressalta que a imprecisão tende a ser maior em solos com valores de coeficientes de atenuação próximos aos da água.

A relação entre coeficientes de atenuação lineares $\left(\mu_{1}\right)$ e UT dos diferentes materiais e a respectiva equação linear são mostradas na Figura 2.

Substituindo o coeficiente angular desta equação em (1), considerando-se que a composição química do solo é espacialmente homogênea, e incluindo a contribuição do teor de água, obteve-se:

$\mathrm{UT}=986.16\left(\mu_{\mathrm{m} \text {-solo }} \cdot \rho_{\text {solo }}+\mu_{\mathrm{m} \text {-água }} \cdot \theta\right)$,

em que: UT é o valor médio de unidades tomográficas, em cada linha (média de cinco pixels), obtidos do mapa tomográfico gerado para cada amostra; $\mu_{\mathrm{m} \text {-solo }}$ $\left(\mathrm{cm}^{2} \mathrm{~g}^{-1}\right)$ é o coeficiente de atenuação em massa do solo; $\rho_{\text {solo }}\left(\mathrm{Mg} \mathrm{m}^{-3}\right)$ é a densidade do solo; $\mu_{\mathrm{m} \text {-água }}$ $\left(\mathrm{cm}^{2} \mathrm{~g}^{-1}\right)$ é o coeficiente de atenuação em massa do líquido (água) e $\theta\left(\mathrm{cm}^{3}\right.$ de $\mathrm{H}_{2} \mathrm{O}$ por $\mathrm{cm}^{3}$ de solo) é a umidade volumétrica.

Com os resultados obtidos dos coeficientes de atenuação em massa (Tabela 1) e dos parâmetros da reta de calibração do minitomógrafo de raios-X e gama, substituiu-se estes valores na equação 4, obtendose as seguintes relações para o cálculo da densidade do solo nos dois horizontes:

Horizonte A: $\mathrm{D}_{\mathrm{s}}=[(\mathrm{UT} / 986,16)-(0,200 . \theta)] / 0,267$;

Horizonte B: $\mathrm{D}_{\mathrm{s}}=[(\mathrm{UT} / 986,16)-(0,200$. $\theta)] / 0,297$. (6)

Deste modo, utilizando amostras indeformadas, é possível quantificar a densidade, em perfis ortogonais à superfície do solo, permitindo a caracterização da densidade desde a superfície até a profundidade analisada e possibilitando que estes dados sejam apresentados em gráficos, com a densidade do solo $\left(\mathrm{Mg} \mathrm{m}^{-3}\right)$ no eixo das abscissas (x) e a profundidade $(\mathrm{m})$ ao longo do perfil do solo no eixo das ordenadas $(\mathrm{y})$.

A confiabilidade (precisão) do equipamento e da configuração adotada na calibração foi avaliada por meio da repetibilidade e da homogeneidade dos dados obtidos de três tomografias da água destilada (substância homogênea) em um recepiente cilíndrico

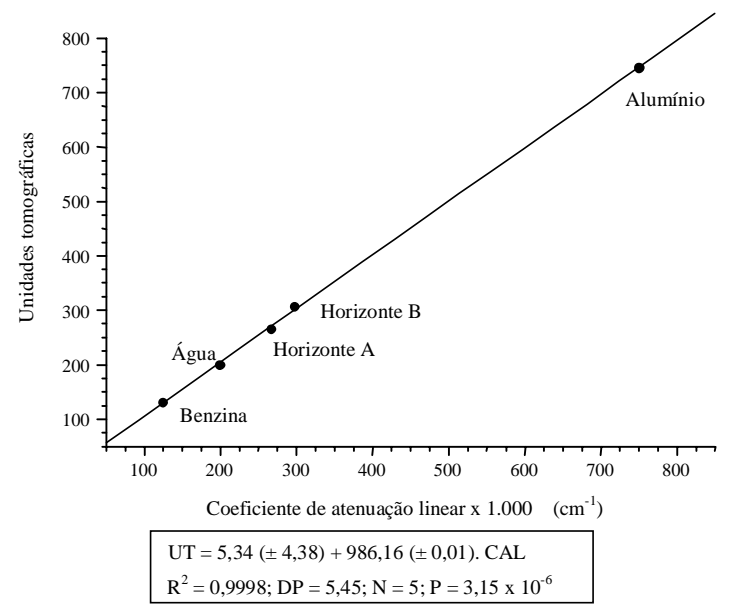

Figura 2. Correlação entre as unidades tomográficas (UT) obtidas pelas tomografias e os coeficientes de atenuação lineares (CAL) medidos nos horizontes A e B do Planossolo e nos materiais analisados.

Pesq. agropec. bras., Brasília, v. 38, n. 7, p. 819-826, jul. 2003 
$\left(\varnothing_{\text {interno }}=98,5 \mathrm{~mm}\right)$. A Tabela 2 mostra a distribuição dos valores centrais ( $7 \times 7$ pixels) das UTs, avaliada pelos parâmetros estatísticos da análise realizada utilizando-se os valores centrais. Os valores estatísticos obtidos demonstram a confiabilidade dos dados fornecidos pelo equipamento, pois a diferença entre os valores da média e da moda representa, nas três tomografias, valor médio menor que $1,7 \%$, indicando baixa variabilidade da análise de substâncias homogêneas. O valor médio obtido do coeficiente de variância nos mapas tomográficos foi aproximadamente $2,7 \%$, ou seja, as variações dos valores de UT foram pequenas, indicando boa homogeneidade. Os valores médios das UTs, quando comparados entre si, mostram variação menor que $0,7 \%$, significando boa repetibilidade dos dados fornecidos pelo equipamento utilizado. Segundo Crestana et al. (1996), a magnitude dos valores do desvio-padrão pode ter sido influenciada por alterações na região analisada ou da espessura dessa região, condições que produzem artefatos na reconstrução da imagem, ou ainda pela interferência de fenômenos como o de Gibbs (quando há interfaces de descontinuidade) e de endurecimento do feixe (microrregiões com maior absorção de baixas energias).

Para a determinação do erro atribuído ao equipamento, estipulou-se a diferença de uma UT e fixou-se o valor de $\theta$ para os diferentes horizontes do Planossolo. Substituindo-se esta diferença nas equações 5 e 6, obteve-se a sensibilidade (Sens.) do equipamento nas condições utilizadas no presente estudo:

Horizonte A: Sens. $=0,0038 \mathrm{Mg} \mathrm{m}^{-3}$ por UT; Horizonte B: Sens. $=0,0034 \mathrm{Mg} \mathrm{m}^{-3}$ por UT.

A média dos valores de UT encontrada nas diferentes amostras foi de aproximadamente 500 UTs.

Tabela 2. Parâmetros estatísticos da região central de três tomografias computadorizadas de substância homogênea (água), realizadas em seqüência de tempo ${ }^{(1)}$.

\begin{tabular}{lrrr}
\hline Parâmetro estatístico & Água 1 & Água 2 & \multicolumn{1}{c}{ Água 3 } \\
\hline Média (UT) & 203,63 & 202,27 & 203,23 \\
Moda (UT) & 198,00 & 198,00 & 203,00 \\
Desvio padrão & 5,37 & 5,55 & 5,58 \\
CV (\%) & 2,64 & 2,74 & 2,75 \\
Variância & 28,86 & 30,78 & 31,14 \\
\hline
\end{tabular}

(1)Água 1: de 14h a 1h10; Água 2: de 1 h13 a 12h23; Água 3: de $13 \mathrm{~h} 30$ a 0h40.
Considerando a variação média de aproximadamente 2,7\% (Tabela 2), o erro médio nas amostras será de aproximadamente $\pm 13,5$ UTs, resultando:

Erro no horizonte A: $\pm 13,5 \times 0,0038= \pm 0,051 \mathrm{Mg} \mathrm{m}^{-3}$; Erro no horizonte B: $\pm 13,5 \times 0,0034= \pm 0,046 \mathrm{Mg} \mathrm{m}^{-3}$.

A diferença entre os valores do erro dos dois horizontes é pequena, evidenciando que as condições de trabalho foram adequadas para a determinação dos coeficientes de atenuação, na energia de 59,6 keV (fonte de ${ }^{241} \mathrm{Am}$ ).

$\mathrm{O}$ erro médio do equipamento é de aproximadamente $0,049 \mathrm{Mg} \mathrm{m}^{-3}$ no solo em estudo. Para diferenciação de valores de densidade do Planossolo, recomenda-se utilizar o Erro equipamento quando o erro do equipamento for maior do que o desvio-padrão da amostra e o desvio-padrão ${ }_{a m o s t r a}$ quando o desvio-padrão da amostra for maior que o erro do equipamento. Leva-se sempre em consideração a pior condição (maior erro). Deste modo, quando se comparam valores médios de densidade deste Planossolo obtidos pela TC, com diferenças entre si de aproximadamente $0,49 \mathrm{Mg} \mathrm{m}^{-3}$, considera-se que não há diferenças significativas entre eles. Ferraz \& Mansell (1979) relatam que mesmo em condições ótimas de operação do sistema de medida de equipamentos, como o utilizado no presente trabalho, são observados valores de desviopadrão de grandeza igual ao erro, ou seja, comportamento igual ao anteriormente mencionado. Tal fato indica que a variação nos valores de densidade do solo estão coerentes com o intervalo aceitável.

Com o objetivo de minimizar os erros inerentes à TC, sugere-se, sempre que possível, fazer várias medidas para cada uma das diferentes situações, de modo a aumentar a confiabilidade e representatividade dos valores médios; checar a calibração em intervalos regulares durante a seqüência de análise das amostras e o valor de $\mathrm{I}_{0}$, também como forma de avaliação da colimação, devendo as suas flutuações não serem maiores que $2 \%$; utilizar medidas durante intervalo de tempo maior; utilizar amostras com espessuras próximas ao ideal (entre 3 e $8 \mathrm{~cm}$ ) para a energia de 59,6 keV nos diferentes solos (Ferraz, 1974).

Outras fontes de raios gama podem ser usadas. No presente estudo optou-se pela fonte de ${ }^{241} \mathrm{Am}$ 
em relação à de ${ }^{137} \mathrm{Cs}$ por causa da necessidade de menor espessura da blindagem da fonte. Chieppe Junior (1993) afirma que $3 \mathrm{~mm}$ de chumbo são suficientes para isolar a radiação emitida pela fonte, tornando o conjunto mais leve e obtendo grande sensibilidade quando empregado em valores de espessura das amostras. No caso de amostras de maior espessura analisadas com o ${ }^{137} \mathrm{Cs}$, os erros certamente seriam maiores $\left(\right.$ Erro $=$ contagens ${ }^{1 / 2} /$ contagens), embora o tempo gasto para análises de amostra de perfil fosse menor porque não necessitaria de seccionamento das amostras.

\section{Conclusões}

1. A técnica da tomografia computadorizada permite a determinação dos valores médios de parâmetros físicos do solo em volumes reduzidos $(0,4 \times 0,4 \times 0,4 \mathrm{~cm})$, e a identificação e localização exata da ocorrência de heterogeneidades (gradiente de densidade ou de umidade).

2. A técnica da tomografia computadorizada apresenta confiabilidade para estudo de parâmetros físicos nos horizontes A e B de Planossolos, pela boa precisão e adaptabilidade proporcionada pelos baixos valores do erro atribuído ao equipamento.

3. A adequada coleta e uso de amostras e a calibração correta do minitomógrafo são fundamentais no emprego da tomografia computadorizada em estudos de parâmetros físicos de Planossolos.

\section{Referências}

ANDERSON, S. H.; HOPMANS, J. W. (Ed.). Tomography of soil-water-root process. Madison: Soil Science Society of America, 1994. 148 p.

APPOLONI, C. R.; CESAREO, R. Microscanning and microtomography with X-ray tubes. Rome: Università degli Studi di Roma La Sapienza-CISB, 1994. 84 p. (Rapport, 04.94).

AYLMORE, L. A. G. Use of computer-assisted tomography in studying water movement around plant roots. Advances in Agronomy, San Diego, v. 49, p. 1-53, 1993

CÁSSARO, F. M. Tomografia de dupla energia para caracterização físico-hídrica de meios porosos deformáveis sob diferentes graus de hidratação. 1994.
194 f. Dissertação (Mestrado em Física) - Universidade de São Paulo, São Carlos, 1994

CHIEPPE JUNIOR, J. B. Estudo e comparação de diferentes métodos de determinação da umidade do solo em laboratório. 103 f. 1993. Dissertação (Mestrado em Agronomia) - Universidade Estadual Paulista, Botucatu, 1993.

COREY, J. C.; PETERSON, S. F.; WAKAT, M. A. Measurement of attenuation of ${ }^{137} \mathrm{Cs}$ and ${ }^{241} \mathrm{Am}$ gamma rays for soil density and water content determinations. Soil Science Society of America Proceedings, Madison, v. 35, p. 215-219, 1971.

CRESTANA, S. A tomografia computadorizada como um novo método para estudos da física da água no solo. 1985.98 f. Tese (Doutorado em Física) - Universidade de São Paulo, São Carlos, 1985.

CRESTANA, S. Noninvasive measurements. In: SPOSITO, G.; REGINATO, R. J. (Ed.). Opportunities in basic soil science research. Madison: Soil Science Society of America, 1992. p. 83-85.

CRESTANA, S.; CRUVINEL, P. E.; MASCARENHAS, S.; BISCEGLI, C. I.; MARTIN NETO, L. M.; COLNAGO, L. A. (Ed.). Instrumentação agropecuária: contribuições no limiar do novo século. Brasília: EmbrapaSPI, 1996. 291 p.

CRESTANA, S.; CRUVINEL, P. E.; VAZ, C. M. P.; CESAREO, R.; MASCARENHAS, S.; REICHARDT, K. Calibração e uso de um tomógrafo computadorizado em ciência do solo. Revista Brasileira de Ciência do Solo, Campinas, v. 16, n. 2, p. 161-167, 1992.

CRUVINEL, P. E. Minitomógrafo de raio $\mathrm{X}$ e raio gama computadorizado para aplicações multidisciplinares. 1987. 325 f. Tese (Doutorado em Engenharia Elétrica) Universidade Estadual de Campinas, Campinas, 1987.

CRUVINEL, P. E.; CESARIO, R.; CRESTANA, S.; MASCARENHAS, S. X and $\gamma$ ray computerized minitomograph scanner for soil science. IEEE Transactions on Instrumentation and Measurement, New York, v. 39 , n. 5 , p. $745-750,1990$.

CRUVINEL, P. E.; COLNAGO, L. A. (Ed.). Advances in agricultural tomography. São Carlos: EmbrapaCNPDIA, 2000. 158 p.

EMBRAPA. Centro Nacional de Pesquisa de Solos (Rio de Janeiro, RJ). Sistema brasileiro de classificação do solo. Brasília: Embrapa-SPI/Embrapa-CNPS, 1999. 412 p. 
ESTADOS UNIDOS. Department of Agriculture. Soil Survey Staff. Keys to soil taxonomy. $4^{\text {th }}$ ed. Blacksburg: Pocahontas, 1990. 422 p. (Soil Management Support Services Technical Monograph, 6).

FERRAZ, E. S. B. Determinação simultânea da densidade e umidade de solos por atenuação de raios gama do ${ }^{241} \mathbf{C s ~ e}^{241} \mathbf{A m}$. 1974. 120 f. Tese (Livre Docência em Física) - Escola Superior de Agricultura Luiz de Queiroz, Piracicaba, 1974.

FERRAZ, E. S. B.; MANSELL, R. S. Determining water content and bulk density of soil by gamma ray attenuation methods. Gainesville: University of Florida, 1979. 51 p. (Technical Bulletin, 807).

IBGE (Rio de Janeiro, RJ). Folha SH.22 Porto Alegre e parte das folhas SH.21 Uruguaiana e SI.22 Lagoa Mirim. Rio de Janeiro, 1986. 796 p. (Levantamento de Recursos Naturais, 33).

MANSEL, R. S.; HAMMOND, L. C.; McCURDY, R. M Coincidence and interference corrections for dual energy gamma ray measurement of soil density and water content. Soil Science Society of America Proceedings, Madison, v. 37 , n. 2 , p. $500-504,1973$.
NAIME, J. M. Projeto e construção de um tomógrafo portátil para estudos de ciência do solo e plantas, em campo. 1994. 87 f. Dissertação (Mestrado em Física) Universidade de São Paulo, São Carlos, 1994.

PEDROTTI, A. Avaliação da compactação de um planossolo submetido a diferentes sistemas de cultivo. 1996. 96 f. Dissertação (Mestrado em Agronomia) Universidade Federal de Pelotas, Pelotas, 1996.

PEDROTTI, A.; PAUlETTO, E. A.; GOMES, A. S.; TURATTI, A. L.; CRESTANA, S. Sistemas de cultivo de arroz irrigado e a compactação de um Planossolo. Pesquisa Agropecuária Brasileira, Brasília, v. 36, n. 4, p. 709-715, abr. 2001.

PINTO, L. F. S.; PAULETTO, E. A.; GOMES, A. S.; SOUSA, R. O. Caracterização de solos de várzea. In: GOMES, A. S.; PAUlETTO, E. A. (Ed.). Manejo de solo e da água em áreas de várzea. Pelotas: EmbrapaCPACT, 1999. p. 11-36.

VAZ, C. M. P. Tomografia computadorizada aplicada a estudos de compactação de solos. 1989. 110 f. Dissertação (Mestrado em Solos e Nutrição de Plantas) - Escola Superior de Agricultura Luiz de Queiroz, Piracicaba, 1989. 